INPLASY

PROTOCOL

To cite: Tang et al.

Acupuncture at local and distant points for cancer pain :A protocol for systematic review and meta-analysis. Inplasy protocol 202080046. doi:

10.37766/inplasy2020.8.0046

Received: 11 August 2020

Published: 11 August 2020

Corresponding author: Zhao Jin

jinzhaocduntcm@163.com

Author Affiliation:

Chengdu University of

Traditional Chinese Medicine

Support: Nos. $2018 S Z 0089$

Review Stage at time of this submission: Preliminary searches.

Conflicts of interest:

No conflict of interest.

\section{Acupuncture at local and distant points for cancer pain: A protocol for systematic review and meta-analysis}

Tang, Y1; Zuo, Y2; Shi, K³ Huang, T4; Zhao, C5; Yan, H6; Jin, Z7.

Review question / Objective: Is the curative effect related to acupoint selection? Compared with sham control or routine care, is the choice of acupuncture points related to the relief of cancer pain?In cancer patients, is the choice of acupuncture points related to the reduction of painkillers? Information sources: From the beginning of its establishment to April 2020, the following electronic databases will be searched, including PubMed, Cochrane Library, EMBASE, Web of Science, WHO International Clinical Trial Registration Platform, China National Knowledge Infrastructure (CNKI), Wanfang Database, Chinese Biomedical Literature Database (CBM), the Chongqing VIP Chinese Science and Technology Journal Database (VIP).

INPLASY registration number: This protocol was registered with the International Platform of Registered Systematic Review and Meta-Analysis Protocols (INPLASY) on 11 August 2020 and was last updated on 11 August 2020 (registration number INPLASY202080046).

\section{INTRODUCTION}

Review question / Objective: Is the curative effect related to acupoint selection? Compared with sham control or routine care, is the choice of acupuncture points related to the relief of cancer pain? In cancer patients, is the choice of acupuncture points related to the reduction of painkillers?

Condition being studied: $40 \%$ of people with early or middle stage cancer and $90 \%$ of those with advanced cancer suffer from moderate to severe pain. Up to $70 \%$ of patients with cancer-related pain cannot get enough pain relief, which affects their 
physical and mental health and reduces their quality of life. Although the analgesic ladder approaches provided by the World Health Organization can effectively relieve cancer pain, the addictive nature of painkillers and the adverse effects of pharmacological interventions pose critical challenges to pain management. In addition, studies have found that long-term use of high-dose anesthetics is easy to promote tumor angiogenesis, accelerate tumor growth, and increase the spread of cancer cells. Because pain is multidimensional, a multidisciplinary and comprehensive approach is needed to successfully control pain. In the United States, government organizations and 14 major organizations in the field, such as the American Society of Clinical Oncology and the National Comprehensive Cancer Network, recommend non-drug interventions such as acupuncture to manage cancer pain. With more and more evidence of the efficacy of acupuncture, most National Cancer Institute-designated comprehensive cancer centers have begun offering acupuncture. In addition, there are guidelines for acupuncture in the treatment of cancer pain.

\section{METHODS}

Participant or population: Participants who use acupuncture to treat cancer pain among aged 18 or older will be included, regardless of their gender, nationality, education, or financial status.

Intervention: Acupuncture,ear acupuncture, electroacupuncture.

Comparator: The comparison could be between sham or placebo acupuncture and analgesic therapy or usual care for managing cancer pain were included.

Study designs to be included: Randomised controlled trials (RCTs) that evaluated any type of invasive acupuncture for pain directly related to cancer in adults aged 18 years or over.

Eligibility criteria: Studies comparing acupuncture with another traditional
Chinese medicine therapy (eg, herbal medicine, massage) were excluded. Studies of acupuncture for short-term analgesia associated with surgical procedures were excluded. Studies that only reported improvement rates were excluded. Non-randomized trials; nonclinical trials. Patients with other coexisting acute or chronic illness; articles not in English or Chinese; articles which data analysis did not fulfill protocol criteria.

Information sources: From the beginning of its establishment to April 2020, the following electronic databases will be searched, including PubMed, Cochrane Library, EMBASE, Web of Science, WHO International Clinical Trial Registration Platform, China National Knowledge Infrastructure (CNKI), Wanfang Database, Chinese Biomedical Literature Database (CBM), the Chongqing VIP Chinese Science and Technology Journal Database (VIP).

Main outcome(s): The primary outcome was pain intensity measured by the Brief Pain Inventory, Numerical Rating Scale, Visual Analog Scale, Verbal Rating Scale, and other validated instruments for assessing the intensity of pain.

Quality assessment / Risk of bias analysis: The risk of bias will be assessed according to the Cochrane Handbook version 5.1 .0 by 2 reviewers. The following factors were assessed: 1. Randomization sequence generation: was the allocation sequence adequately generated? 2. Treatment allocation concealment: was the allocated treatment adequately concealed from study participants and clinicians and other healthcare or research staff at the enrollment stage? 3 . Blinding: were the personnel assessing outcomes and analyzing data sufficiently blinded to the intervention allocation throughout the trial? 4. Completeness of outcome data: were participant exclusions, attrition, and incomplete outcome data adequately addressed in the published report? 5 . Evidence of selective outcome reporting, and might this have affected the study results? 6. Other sources of bias: was the trail apparently free of any other problems 
that could produce a high risk of bias? Disagreements were solved by discussion until a consensus was reached.

Strategy of data synthesis: Data analysis will be performed with RevManV.5.3 software provided by the Cochrane collaboration. Effective Rate was calculated by relative risk, and the HAMA score will be calculated by mean difference. Heterogeneity is recognized as significant when $12 \geq 50 \%$. A fixed-effect model will be performed when there is no significant heterogeneity, otherwise a random-effects model will be performed.

Subgroup analysis: We intend to explore clinical heterogeneity by testing the effect of acupoint selection on the efficacy of acupuncture in the treatment of cancer pain. The grouping will be carried out according to the location of acupoints (local acupoints, distant acupoints, local and distant acupoints) and the number of acupoints. If the previous analysis shows considerable heterogeneity, we will conduct a further subgroup analysis.

Sensibility analysis: Sensitivity analysis will also be employed to explore possible factors that may lead to heterogeneity, such as intervention measures (electroacupuncture and manual acupuncture),control measures, length of treatment or quality of articles, etc. If quantitative synthesis is not appropriate, we will conduct a narrative synthesis.

Language: English and Chinese.

Country(ies) involved: China.

Keywords: Acupuncture, local and distant acupoints, cancer pain, protocol, a systematic review.

Contributions of each author:

Author 1 - Ying Tang - Writing-review \& editing.

Author 2 - Yuling Zuo - Methodology.

Author 3 - Kejin Shi - Writing-original draft.

Author 4 - Teng Huang - Data curation.

Author 5 - Chenghao Zhao - Project administration.
Author 6 - Hang Yan - Software.

Author 7 - Zhao Jin - Supervision. 